\title{
Formation of ternary inclusion crystal and enantioseparation of alkyl aryl sulfoxides by the salt of urea-modified L-phenylalanine and an achiral amine
}

Koichi Kodama*, Rina Morita and Takuji Hirose

Graduate School of Science and Engineering, Saitama University

255 Shimo-ohkubo, Sakura-ku, Saitama City, Saitama 338-8570, Japan

Table of Contents

Synthesis and characterization

S2-S5

Crystallographic data of the reported compounds (Table S1) S6 


\section{Synthesis and characterization}

$N$-(Phenylcarbamoyl)-L-phenylalanine (1a). To a solution of L-phenylalanine (0.363 g, 2.20 $\mathrm{mmol}$ ) in $0.25 \mathrm{M}$ aqueous $\mathrm{NaHCO}_{3}$ solution $(100 \mathrm{~mL})$, phenyl isocyanate $(0.236 \mathrm{~g}, 1.98 \mathrm{mmol})$ was added and the mixture was stirred for $4 \mathrm{~h}$ at room temperature. The mixture was acidified by the addition of $1 \mathrm{M}$ aqueous $\mathrm{HCl}$ solution and extracted with ethyl acetate $(100 \mathrm{~mL} \times 3)$. The organic phase was dried over anhydrous magnesium sulfate and the solvent was removed under reduced pressure. The residue was washed with methylene chloride to afford the product 1a $(0.365 \mathrm{~g}, 1.28$ mmol, 64\%) as a white solid.

Mp: $157.8-159.5{ }^{\circ} \mathrm{C} .[\alpha]_{\mathrm{D}}=+95.3(\mathrm{c} 0.991, \mathrm{EtOH}) .{ }^{1} \mathrm{H}$ NMR $\left(400 \mathrm{MHz}, \mathrm{DMSO}-d_{6}\right): \delta 8.69(\mathrm{~s}, 1 \mathrm{H})$, 7.38-7.26 (m, 4H), 7.26-7.17 (m, 5H), 6.89 (t, $J=7.4 \mathrm{~Hz}, 1 \mathrm{H}), 6.34(\mathrm{~d}, J=8.0 \mathrm{~Hz}, 1 \mathrm{H}), 4.50-4.42(\mathrm{~m}$, $1 \mathrm{H}), 3.09\left(\mathrm{dd}, J_{1}=13.6 \mathrm{~Hz}, J_{2}=5.2 \mathrm{~Hz}, 1 \mathrm{H}\right), 2.95\left(\mathrm{dd}, J_{1}=13.6 \mathrm{~Hz}, J_{2}=7.4 \mathrm{~Hz}, 1 \mathrm{H}\right) .{ }^{13} \mathrm{C} \mathrm{NMR}$ (100 MHz, DMSO-d6): $\delta$ 173.4, 154.5, 140.1, 137.1, 129.2, 128.6, 128.1, 126.4, 121.1, 117.4, 53.5,a 37.3. IR (KBr): $v_{\max } 3332,1702,1650,1597,1551,1497,1444,1236 \mathrm{~cm}^{-1}$. MS (MALDI-TOF, matrix; gentisic acid): $\mathrm{m} / \mathrm{z}$ calcd for $[\mathrm{M}+\mathrm{Na}]^{+}: 307.105$, found: 307.110 .

To a solution of $1 \mathbf{a}(24.0 \mathrm{mg}, 0.0840 \mathrm{mmol})$ in toluene $(8 \mathrm{ml})$ and methanol $(2 \mathrm{ml})$ was added $\mathrm{TMSCHN}_{2}$ (2.0 M solution in hexane) until the solution remained yellow. The mixture was stirred for $10 \mathrm{~min}$ at ambient temperature and the solvent was removed under reduced pressure. The residue was purified by silica gel PTLC (chloroform/ethyl acetate $=9: 1$ ) to quantitatively produce methyl ester of 1a as a colorless liquid. The ee value was determined by chiral HPLC analysis (ChiralCel OD-3, 10\% IPA in hexane, $1.0 \mathrm{ml} / \mathrm{min}, 254 \mathrm{~nm}$ UV detector). Retention times: $t_{\mathrm{r}}=20 \mathrm{~min}$ for $(S)$-isomer and $t_{\mathrm{r}}=26 \mathrm{~min}$ for $(R)$-isomer. ${ }^{1} \mathrm{H} \mathrm{NMR}\left(300 \mathrm{MHz}, \mathrm{CDCl}_{3}\right): \delta 7.40-7.18(\mathrm{~m}, 10 \mathrm{H}), 6.34$ $(\mathrm{s}, 1 \mathrm{H}), 5.15(\mathrm{~d}, J=3.9 \mathrm{~Hz}, 1 \mathrm{H}), 4.85-4.79(\mathrm{~m}, 1 \mathrm{H}), 3.74(\mathrm{~s}, 3 \mathrm{H}), 3.18\left(\mathrm{dd}, J_{1}=13.9 \mathrm{~Hz}, J_{2}=5.9 \mathrm{~Hz}\right.$, $1 \mathrm{H}), 3.09\left(\mathrm{dd}, J_{1}=13.9 \mathrm{~Hz}, J_{2}=5.9 \mathrm{~Hz}, 1 \mathrm{H}\right)$.

$N$-(Benzylcarbamoyl)-L-phenylalanine (1b). To a solution of triphosgene (0.590 g, $1.99 \mathrm{mmol})$ in dry toluene $(25 \mathrm{ml})$ was added benzylamine $(0.406 \mathrm{~g}, 3.79 \mathrm{mmol})$ in dry toluene $(10 \mathrm{ml})$ dropwise under $\mathrm{N}_{2}$ atmosphere and the mixture was refluxed for $5 \mathrm{~h}$. After cooling to room temperature, the solvent was removed under reduced pressure to afford crude benzyl isocyanate $(0.486 \mathrm{~g})$ as a yellow liquid.

To a solution of L-phenylalanine $(0.693 \mathrm{~g}, 4.19 \mathrm{mmol})$ in $0.25 \mathrm{M}$ aqueous $\mathrm{NaHCO}_{3}$ solution (160 $\mathrm{mL})$, benzyl isocyanate $(0.486 \mathrm{~g})$ was added and the mixture was stirred for $14 \mathrm{~h}$ at room temperature. The mixture was acidified by the addition of $1 \mathrm{M}$ aqueous $\mathrm{HCl}$ solution and extracted with chloroform. The organic phase was dried over anhydrous sodium sulfate and the solvent was removed under reduced pressure. The residue was washed with chloroform to afford the product $\mathbf{1 b}$ $(0.127 \mathrm{~g}, 0.425 \mathrm{mmol}, 11 \%)$ as a white solid. 
Mp: $118.0-120.0{ }^{\circ} \mathrm{C} .[\alpha]_{\mathrm{D}}=+40.3\left(c\right.$ 1.01, EtOH). ${ }^{1} \mathrm{H}$ NMR $\left(400 \mathrm{MHz}, \mathrm{DMSO}-d_{6}\right): \delta$ 7.35-7.25 (m, $4 \mathrm{H}), 7.25-7.15(\mathrm{~m}, 6 \mathrm{H}), 6.55(\mathrm{t}, J=6.2 \mathrm{~Hz}, 1 \mathrm{H}), 6.16(\mathrm{~d}, J=8.4 \mathrm{~Hz}, 1 \mathrm{H}), 4.45-4.35(\mathrm{~m}, 1 \mathrm{H}), 4.18(\mathrm{~d}$, $J=6.0 \mathrm{~Hz}, 2 \mathrm{H}), 3.03\left(\mathrm{dd}, J_{1}=13.6 \mathrm{~Hz}, J_{2}=5.2 \mathrm{~Hz}, 1 \mathrm{H}\right), 2.88\left(\mathrm{dd}, J_{1}=13.6 \mathrm{~Hz}, J_{2}=8.0 \mathrm{~Hz}, 1 \mathrm{H}\right)$. ${ }^{13} \mathrm{C}$ NMR (100 MHz, DMSO-d $)$ : $\delta 173.8,157.4,140.5,137.4,129.2,128.1,128.0,126.8,126.4$, 126.3, 53.7, 42.6, 37.5. IR (KBr): $v_{\max }$ 3316, 1717, 1568, 1256, $1062 \mathrm{~cm}^{-1}$. MS (MALDI-TOF, matrix; gentisic acid): $\mathrm{m} / \mathrm{z}$ calcd for $[\mathrm{M}+\mathrm{Na}]^{+}: 321.121$, found: 321.130 .

$N$-((S)- $\alpha$-Methylbenzylcarbamoyl)-L-phenylalanine (1c). To a solution of triphosgene $(0.580 \mathrm{~g}$, $1.95 \mathrm{mmol})$ in dry toluene $(20 \mathrm{ml})$ was added $(S)$-1-phenylethylamine $(0.488 \mathrm{~g}, 4.03 \mathrm{mmol})$ in dry toluene $(10 \mathrm{ml})$ dropwise under $\mathrm{N}_{2}$ atmosphere and the mixture was refluxed for $5 \mathrm{~h}$. After cooling to room temperature, the solvent was removed under reduced pressure to afford crude $(S)$ - $\alpha$-methylbenzyl isocyanate $(0.587 \mathrm{~g})$ as a yellow liquid.

To a solution of L-phenylalanine $(0.663 \mathrm{~g}, 4.01 \mathrm{mmol})$ in $0.25 \mathrm{M}$ aqueous $\mathrm{NaHCO}_{3}$ solution (140 $\mathrm{mL}),(S)$ - $\alpha$-methylbenzyl isocyanate $(0.587 \mathrm{~g})$ was added and the mixture was stirred for $19 \mathrm{~h}$ at room temperature. The mixture was acidified by the addition of $1 \mathrm{M}$ aqueous $\mathrm{HCl}$ solution and extracted with chloroform. The organic phase was dried over anhydrous sodium sulfate and the solvent was removed under reduced pressure. The residue was washed with chloroform to afford the product $1 \mathrm{c}(0.291 \mathrm{~g}, 0.930 \mathrm{mmol}, 23 \%)$ as a white solid.

Mp: 172.0-174.0 ${ }^{\circ} \mathrm{C} .[\alpha]_{\mathrm{D}}=+56.0(c 1.000, \mathrm{EtOH}) .{ }^{1} \mathrm{H}$ NMR $\left(300 \mathrm{MHz}, \mathrm{DMSO}-d_{6}\right): \delta 7.40-7.20(\mathrm{~m}$, $8 \mathrm{H}), 7.20-7.12(\mathrm{~m}, 2 \mathrm{H}), 6.64(\mathrm{~d}, J=8.1 \mathrm{~Hz}, 1 \mathrm{H}), 6.04(\mathrm{~d}, J=8.1 \mathrm{~Hz}, 1 \mathrm{H}), 4.80-4.65(\mathrm{~m}, 1 \mathrm{H})$, 4.44-4.32 (m, 1H), $3.03\left(\mathrm{dd}, J_{1}=13.7 \mathrm{~Hz}, J_{2}=5.3 \mathrm{~Hz}, 1 \mathrm{H}\right), 2.89\left(\mathrm{dd}, J_{1}=13.7 \mathrm{~Hz}, J_{2}=7.4 \mathrm{~Hz}, 1 \mathrm{H}\right)$, $1.32(\mathrm{~d}, J=6.9 \mathrm{~Hz}, 3 \mathrm{H}) .{ }^{13} \mathrm{C}$ NMR (100 MHz, DMSO- $\left.d_{6}\right): \delta 173.7,156.5,145.5,137.1,129.2,128.1$, 128.0, 126.3, 125.6, 53.5, 48.4, 37.5, 23.2. IR (KBr): $v_{\max } 3389,3281,1704,1610,1549,1440,1281$, 1193, 764, $703 \mathrm{~cm}^{-1}$. MS (MALDI-TOF, matrix; gentisic acid): $\mathrm{m} / z$ calcd for $[\mathrm{M}+\mathrm{Na}]^{+}: 335.137$, found: 335.154 .

$N$-((R)- $\boldsymbol{\alpha}$-Methylbenzylcarbamoyl)-L-phenylalanine (1d). To a solution of triphosgene (1.19 g, $4.00 \mathrm{mmol})$ in dry toluene $(40 \mathrm{ml})$ was added $(R)$-1-phenylethylamine $(0.969 \mathrm{~g}, 8.00 \mathrm{mmol})$ in dry toluene $(20 \mathrm{ml})$ dropwise under $\mathrm{N}_{2}$ atmosphere and the mixture was refluxed for $5 \mathrm{~h}$. After cooling to room temperature, the solvent was removed under reduced pressure to afford crude $(S)$ - $\alpha$-methylbenzyl isocyanate $(1.13 \mathrm{~g})$ as a yellow liquid.

To a solution of L-phenylalanine $(1.32 \mathrm{~g}, 8.01 \mathrm{mmol})$ in $0.25 \mathrm{M}$ aqueous $\mathrm{NaHCO}_{3}$ solution $(350 \mathrm{~mL})$, $(R)$ - $\alpha$-methylbenzyl isocyanate $(1.13 \mathrm{~g})$ was added and the mixture was stirred for $15 \mathrm{~h}$ at room temperature. The mixture was acidified by the addition of $1 \mathrm{M}$ aqueous $\mathrm{HCl}$ solution and extracted with chloroform. The organic phase was dried over anhydrous sodium sulfate and the solvent was removed under reduced pressure. The residue was washed with chloroform to afford the product 1d (1.09 g, $3.48 \mathrm{mmol}, 44 \%)$ as a white solid.

Mp: $168.5-169.8^{\circ} \mathrm{C} .[\alpha]_{\mathrm{D}}=+51.1(c 0.978, \mathrm{EtOH}) .{ }^{1} \mathrm{H}$ NMR $\left(400 \mathrm{MHz}, \mathrm{DMSO}-d_{6}\right): \delta 7.35-7.12(\mathrm{~m}$, 
$10 \mathrm{H}), 6.59(\mathrm{~d}, J=8.4 \mathrm{~Hz}, 1 \mathrm{H}), 6.00(\mathrm{~d}, J=8.0 \mathrm{~Hz}, 1 \mathrm{H}), 4.76-4.65(\mathrm{~m}, 1 \mathrm{H}), 4.40-4.28(\mathrm{~m}, 1 \mathrm{H}), 3.00$ $\left(\mathrm{dd}, J_{1}=13.6 \mathrm{~Hz}, J_{2}=5.2 \mathrm{~Hz}, 1 \mathrm{H}\right), 2.87\left(\mathrm{dd}, J_{1}=13.8 \mathrm{~Hz}, J_{2}=7.4 \mathrm{~Hz}, 1 \mathrm{H}\right), 1.28(\mathrm{~d}, J=6.8 \mathrm{~Hz}, 3 \mathrm{H})$. ${ }^{13} \mathrm{C}$ NMR (100 MHz, DMSO-d $\left.d_{6}\right): \delta 173.7,156.6,145.4,137.3,129.2,128.1,126.3,126.3,125.6$, 53.7, 48.4, 37.6, 23.3. IR (KBr): $v_{\max } 3422,3282,1699,1551,1454,1260,1215,762,701 \mathrm{~cm}^{-1} . \mathrm{MS}$ (MALDI-TOF, matrix; gentisic acid): $\mathrm{m} / z$ calcd for $[\mathrm{M}+\mathrm{Na}]^{+}: 335.137$, found: 335.160 .

$N$-(Phenylcarbamoyl)-L-phenylglycine (1e). To a solution of L-phenylglycine (0.333 g, 2.21 $\mathrm{mmol}$ ) in $0.25 \mathrm{M}$ aqueous $\mathrm{NaHCO}_{3}$ solution $(140 \mathrm{~mL})$, phenyl isocyanate $(0.238 \mathrm{~g}, 2.00 \mathrm{mmol})$ was added and the mixture was stirred for $8 \mathrm{~h}$ at room temperature. The mixture was acidified by the addition of $1 \mathrm{M}$ aqueous $\mathrm{HCl}$ solution and extracted with ethyl acetate $(100 \mathrm{~mL} \times 3)$. The organic phase was dried over anhydrous magnesium sulfate and the solvent was removed under reduced pressure. The residue was washed with chloroform to afford the product 1e $(0.361 \mathrm{~g}, 1.34 \mathrm{mmol}$, $67 \%)$ as a white solid.

Mp: $150.0-152.0{ }^{\circ} \mathrm{C} .[\alpha]_{\mathrm{D}}=+159(c 0.602, \mathrm{EtOH}) .{ }^{1} \mathrm{H}$ NMR $\left(400 \mathrm{MHz}, \mathrm{DMSO}-d_{6}\right): \delta 8.69(\mathrm{~s}, 1 \mathrm{H})$, 7.45-7.30 (m, 7H), 7.26-7.16 (m, 2H), $7.00(\mathrm{~d}, J=7.6 \mathrm{~Hz}, 1 \mathrm{H}), 6.93-6.86(\mathrm{~m}, 1 \mathrm{H}), 5.25(\mathrm{~d}, J=7.2$ $\mathrm{Hz}, 1 \mathrm{H}) .{ }^{13} \mathrm{C}$ NMR $\left(100 \mathrm{MHz}, \mathrm{DMSO}-d_{6}\right): \delta 172.4,154.1,139.9,138.1,128.6,128.5,127.8,127.0$, 121.2, 117.4, 56.5. IR (KBr): $v_{\max }$ 2924, 1735, 1654, 1599, 1542, 1497, 754, $694 \mathrm{~cm}^{-1}$. MS (MALDI-TOF, matrix; gentisic acid): $\mathrm{m} / z$ calcd for $[\mathrm{M}+\mathrm{Na}]^{+}: 293.090$, found: 293.113.

$N$-(Benzylcarbamoyl)-L-phenylglycine (1f). To a solution of triphosgene (1.22 g, $4.12 \mathrm{mmol})$ in dry toluene $(40 \mathrm{ml})$ was added benzylamine $(0.871 \mathrm{~g}, 8.13 \mathrm{mmol})$ in dry toluene $(20 \mathrm{ml})$ dropwise under $\mathrm{N}_{2}$ atmosphere and the mixture was refluxed for $4 \mathrm{~h}$. After cooling to room temperature, the solvent was removed under reduced pressure to afford crude benzyl isocyanate $(0.971 \mathrm{~g})$ as a yellow liquid.

To a solution of L-phenylglycine $(0.843 \mathrm{~g}, 5.58 \mathrm{mmol})$ in $0.25 \mathrm{M}$ aqueous $\mathrm{NaHCO}_{3}$ solution (420 $\mathrm{mL})$, benzyl isocyanate $(0.971 \mathrm{~g})$ was added and the mixture was stirred for $23 \mathrm{~h}$ at room temperature. The mixture was acidified by the addition of $1 \mathrm{M}$ aqueous $\mathrm{HCl}$ solution and extracted with chloroform. The organic phase was dried over anhydrous sodium sulfate and the solvent was removed under reduced pressure. The residue was washed with chloroform to afford the product $\mathbf{1 f}$ (0.456 g, $1.60 \mathrm{mmol}, 29 \%)$ as a white solid.

Mp: $156.0-157.5^{\circ} \mathrm{C} .[\alpha]_{\mathrm{D}}=+103(c 0.912, \mathrm{EtOH}) .{ }^{1} \mathrm{H}$ NMR (400 MHz, DMSO- $\left.d_{6}\right): \delta$ 7.42-7.26 (m, $7 \mathrm{H}), 7.26-7.17(\mathrm{~m}, 3 \mathrm{H}), 6.79(\mathrm{~d}, J=8.0 \mathrm{~Hz}, 1 \mathrm{H}), 6.59$ (t, $J=6.0 \mathrm{~Hz}, 1 \mathrm{H}), 5.21(\mathrm{~d}, J=8.0 \mathrm{~Hz}, 1 \mathrm{H})$, 4.28-4.14 (m, 2H). ${ }^{13} \mathrm{C}$ NMR (100 MHz, DMSO- $\left.d_{6}\right): \delta 172.7,157.1,140.4,138.4,128.4,128.1$, 127.7, 127.0, 126.9, 126.5, 56.8, 42.7. IR (KBr): $v_{\max } 3357,1714,1628,1567,1225,696 \mathrm{~cm}^{-1} . \mathrm{MS}$ (MALDI-TOF, matrix; gentisic acid): $\mathrm{m} / \mathrm{z}$ calcd for $[\mathrm{M}+\mathrm{Na}]^{+}: 307.105$, found: 307.140 .

$N$-(Phenylthiocarbamoyl)-L-phenylalanine (1g). To a solution of L-phenylalanine (1.08 g, 6.53 $\mathrm{mmol}$ ) in $0.25 \mathrm{M}$ aqueous $\mathrm{NaHCO}_{3}$ solution $(300 \mathrm{~mL})$, phenyl isothiocyanate $(0.812 \mathrm{~g}, 6.01 \mathrm{mmol})$ 
was added and the mixture was stirred for $21 \mathrm{~h}$ at room temperature. The mixture was acidified by the addition of $1 \mathrm{M}$ aqueous $\mathrm{HCl}$ solution and extracted with ethyl acetate $(100 \mathrm{~mL} \times 3)$. The organic phase was dried over anhydrous magnesium sulfate and the solvent was removed under reduced pressure. The residue was dissolved in small amount of hot dichloromethane and hexane was added to afford the product $1 \mathrm{~g}(0.533 \mathrm{~g}, 1.84 \mathrm{mmol}, 31 \%)$ as a white solid.

Mp: $182.0-183.0{ }^{\circ} \mathrm{C} .[\alpha]_{\mathrm{D}}=-20.3(c 0.994, \mathrm{EtOH}) .{ }^{1} \mathrm{H}$ NMR $\left(400 \mathrm{MHz}, \mathrm{DMSO}-d_{6}\right): \delta 10.58(\mathrm{~s}, 1 \mathrm{H})$, 7.43-7.16 (m, 8H), 6.82-6.74 (m, 2H), 4.80-4.74 (m, 1H), $3.12(\mathrm{~d}, J=4.4 \mathrm{~Hz}, 2 \mathrm{H}) .{ }^{13} \mathrm{C}$ NMR $(100$ MHz, DMSO-d6): $\delta 182.1,173.3,134.3,133.1,129.7,128.5,128.4,128.3,128.0,127.0,60.0,36.0$. IR (KBr): $v_{\max } 3161,1751,1520,1273,1191,738,691 \mathrm{~cm}^{-1}$. MS (MALDI-TOF, matrix; gentisic acid): $\mathrm{m} / z$ calcd for $[\mathrm{M}+\mathrm{Na}]^{+}: 323.082$, found: 323.093 .

$N$-(Phenylacetyl)-L-phenylalanine (1h). To a solution of L-phenylalanine $(0.166 \mathrm{~g}, 1.00 \mathrm{mmol})$ in $0.2 \mathrm{M}$ aqueous $\mathrm{NaOH}$ solution $(15 \mathrm{~mL})$, phenylacetyl chloride $(0.170 \mathrm{~g}, 1.10 \mathrm{mmol})$ was added and the mixture was stirred for $2.5 \mathrm{~h}$ at room temperature. The mixture was acidified by the addition of $1 \mathrm{M}$ aqueous $\mathrm{HCl}$ solution. The precipitate was filtered and washed with water to afford the product 1h $(0.183 \mathrm{~g}, 0.647 \mathrm{mmol}, 65 \%)$ as a white solid.

Mp: $140.0-142.0{ }^{\circ} \mathrm{C} .[\alpha]_{\mathrm{D}}=+28.0(c 1.000, \mathrm{EtOH}) .{ }^{1} \mathrm{H}$ NMR $\left(400 \mathrm{MHz}, \mathrm{CDCl}_{3}\right): \delta$ 7.36-7.10 (m, $8 \mathrm{H}), 6.96-6.90(\mathrm{~m}, 2 \mathrm{H}), 5.83(\mathrm{~d}, J=7.2 \mathrm{~Hz}, 1 \mathrm{H}), 4.85-4.76(\mathrm{~m}, 1 \mathrm{H}), 3.55(\mathrm{~s}, 2 \mathrm{H}), 3.13\left(\mathrm{dd}, J_{1}=14.0\right.$ $\left.\mathrm{Hz}, J_{2}=5.2 \mathrm{~Hz}, 1 \mathrm{H}\right), 3.01\left(\mathrm{dd}, J_{1}=14.0 \mathrm{~Hz}, J_{2}=6.8 \mathrm{~Hz}, 1 \mathrm{H}\right) .{ }^{13} \mathrm{C} \mathrm{NMR}\left(100 \mathrm{MHz}, \mathrm{CDCl}_{3}\right): \delta 173.9$, $171.7,135.5,133.9,129.5,129.2,129.1,128.7,127.6,127.2,53.2,43.4,36.9$. IR (KBr): $v_{\max } 3587$, 3432, 3257, 3106, 1744, 1646, 1577, 1190, 1116, 731, $699 \mathrm{~cm}^{-1}$. MS (MALDI-TOF, matrix; gentisic acid): $m / z$ calcd for $[\mathrm{M}+\mathrm{Na}]^{+}: 306.110$, found: 306.163 . 
Table S1. Summary of crystallographic data reported in this study.

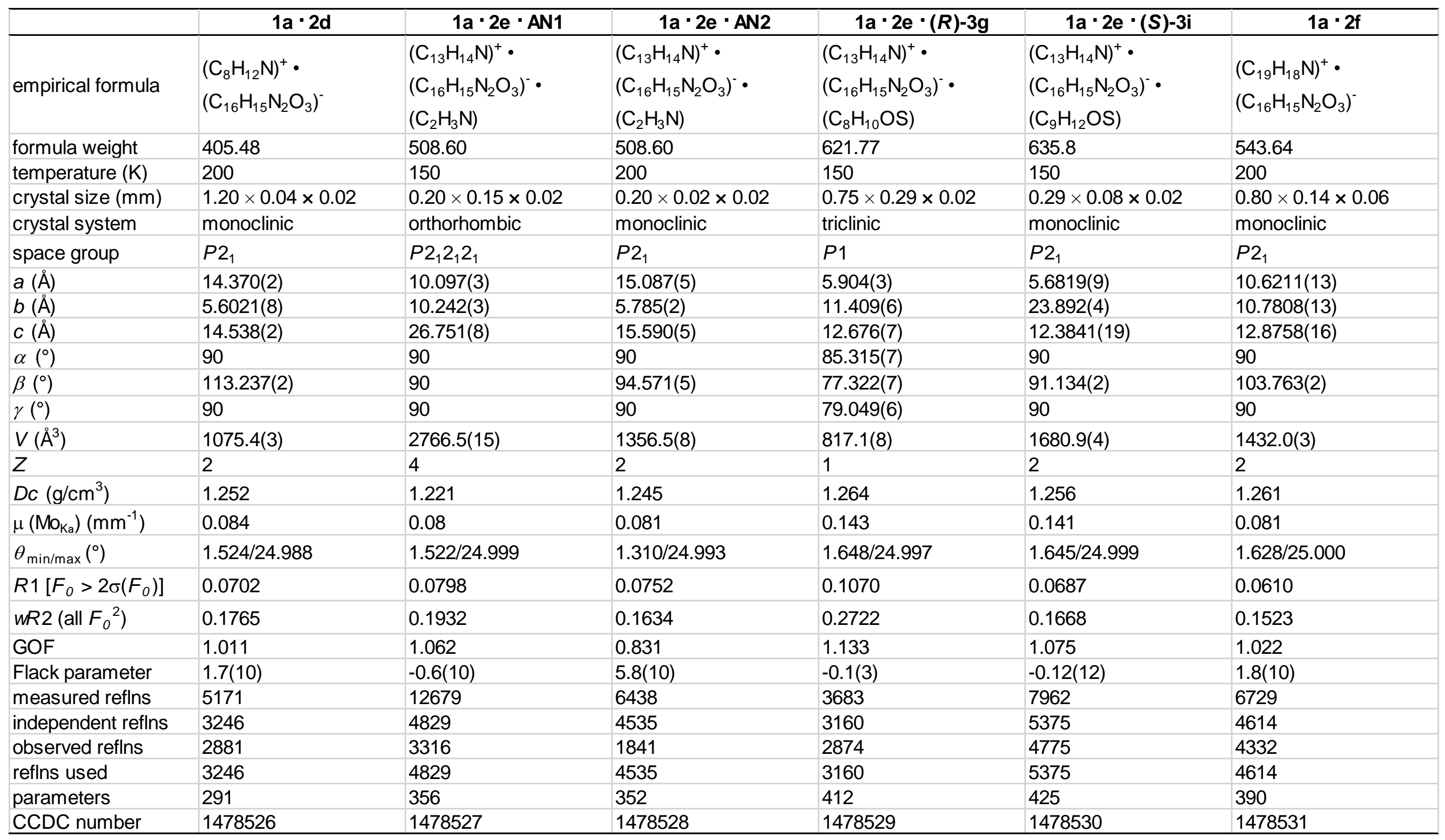




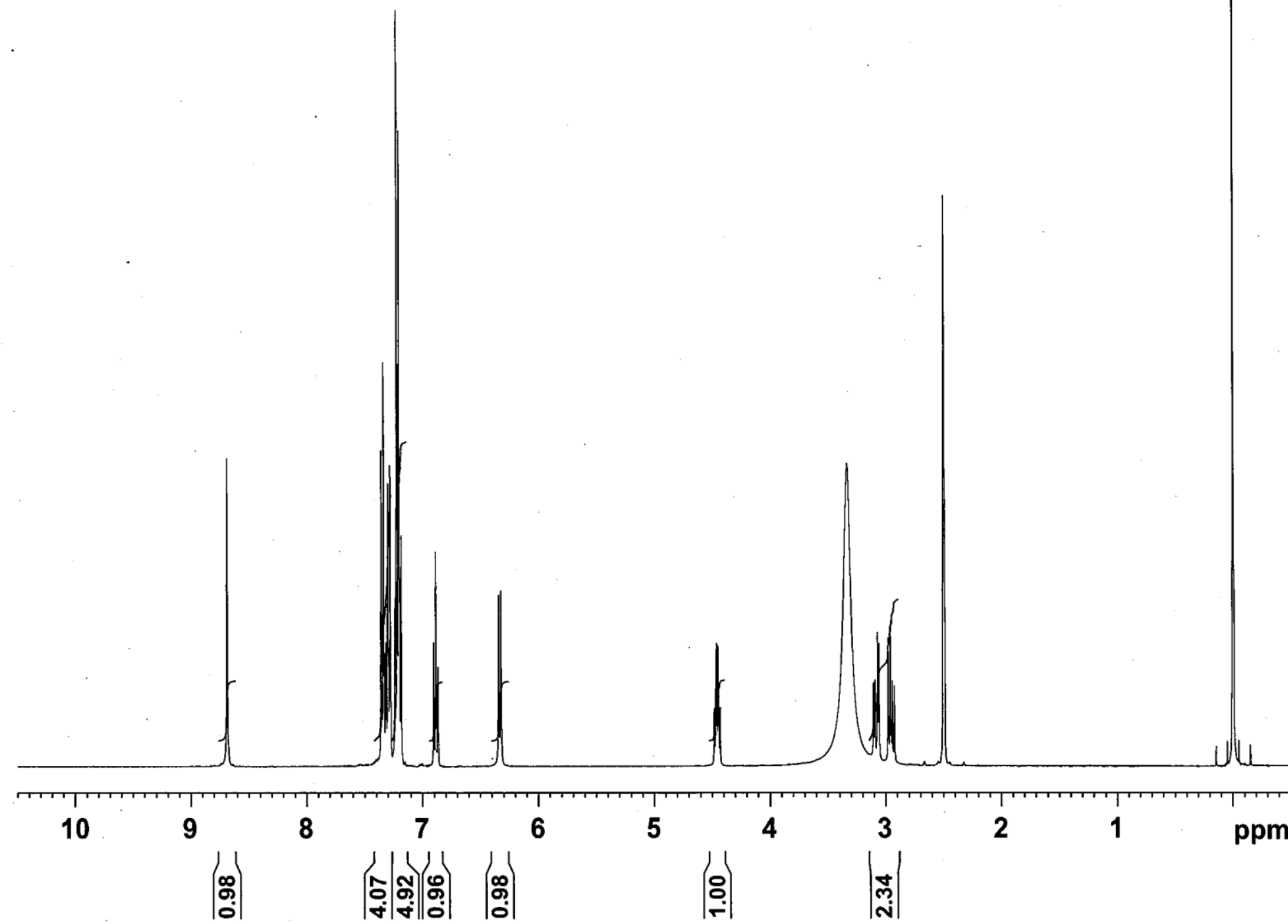

Current Data Parameters

28

F2 - Acquisition Parameters

Date_ 20160723

Time

15.23
20160723

INSTRUM

PROBHD $5 \mathrm{~mm}$ CPONP $1 \mathrm{H} /$

PULPROG $\quad \mathrm{zg} 30$

TD

SOLVENT $\quad 65536$

NS

SWH

$8305.647 \mathrm{~Hz}$

$0.126734 \mathrm{~Hz}$

$\mathrm{AQ}$

$3.9453173 \mathrm{sec}$

$$
10.03
$$

DW 60.200 usec

$\mathrm{DE} \quad 10.00$ usec

TE $300.0 \mathrm{~K}$

D1 1.00000000

$=======$ CHANNEL $\mathrm{f}]$

NUC1

$\mathrm{f} 1$

sec

15.00 usec

$5.1999981 \mathrm{~W}$

F2 - Processing parameters

ST - Processing parameter

SF $\quad 400.1300038 \mathrm{MHz}$

$\begin{array}{lr}\text { SF } & 400.1300038 \\ \text { WDW } & \text { EM }\end{array}$

SSB $\quad 0$

$0.30 \mathrm{~Hz}$

PC 1.00

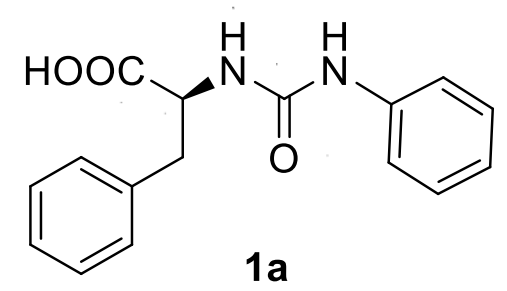


13C with dec. CPQNP

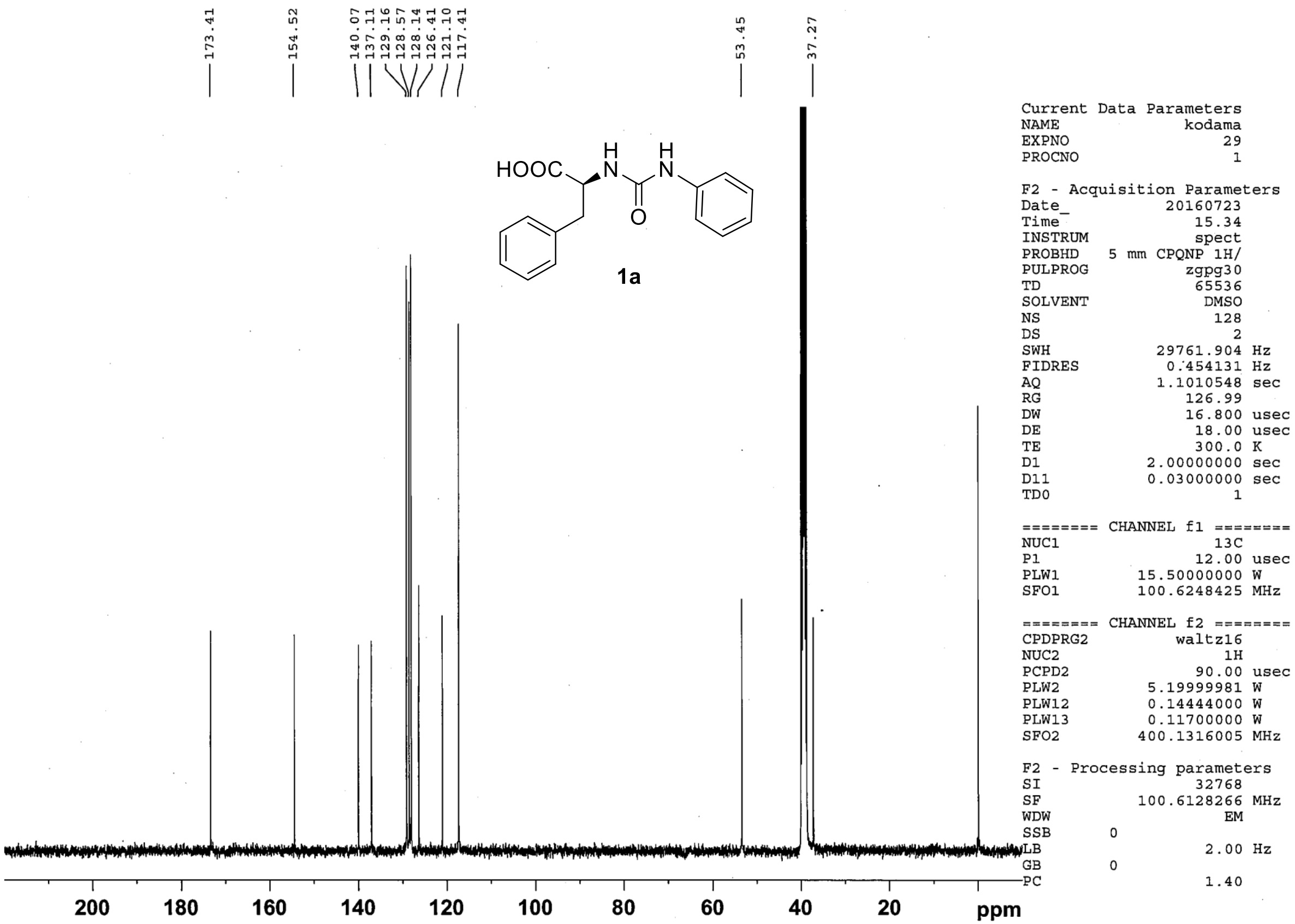




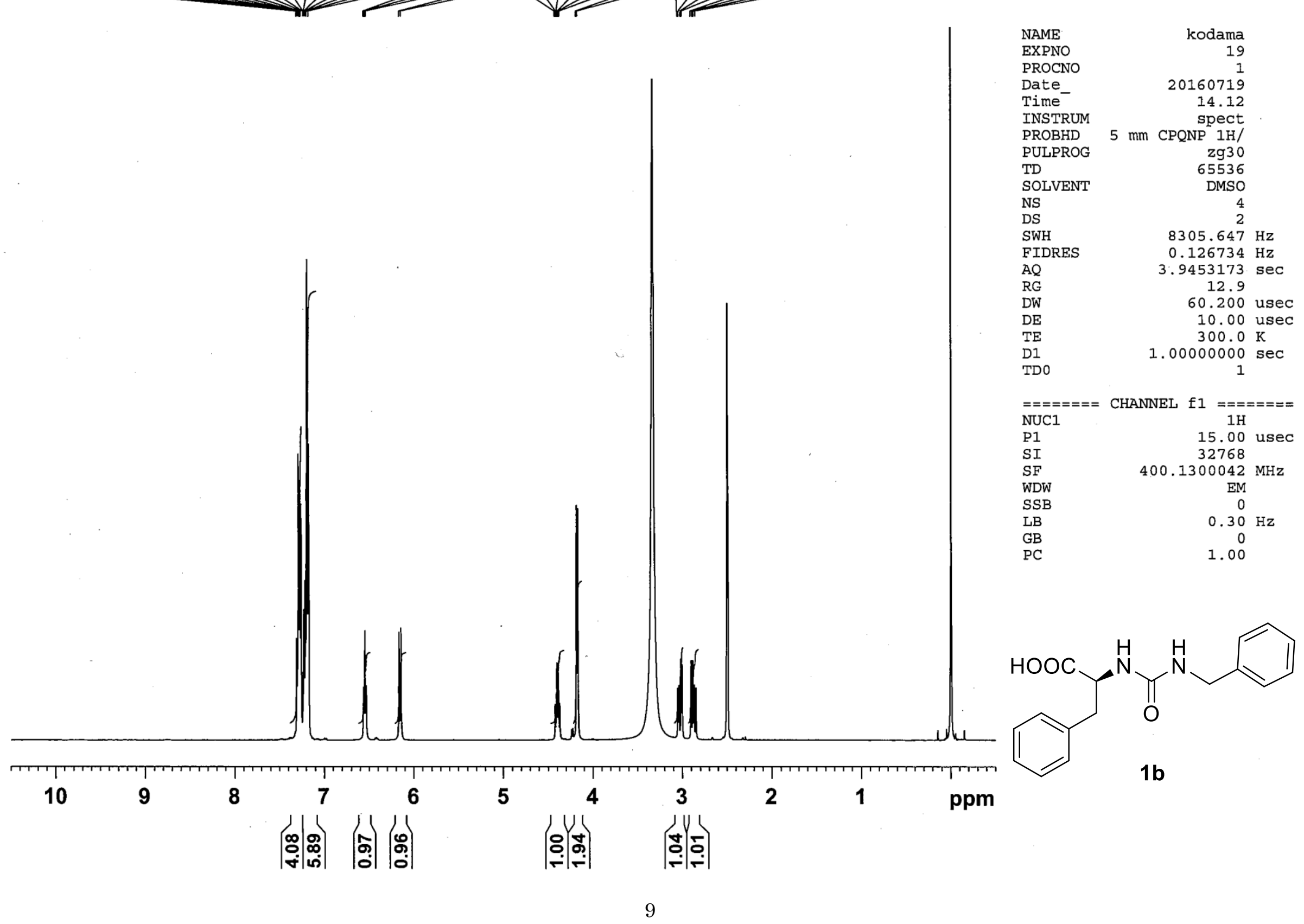


13C with dec. CPQNP

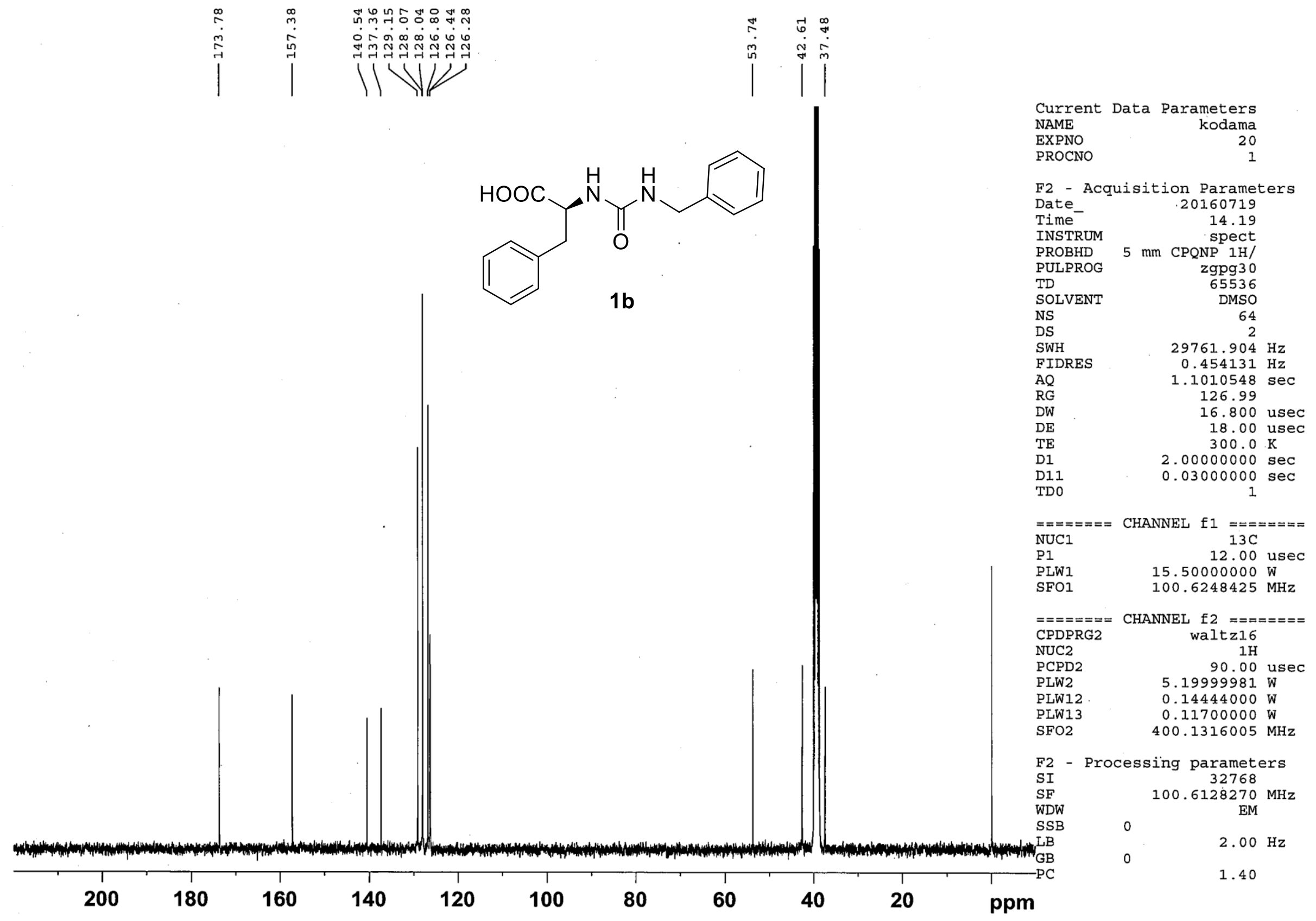




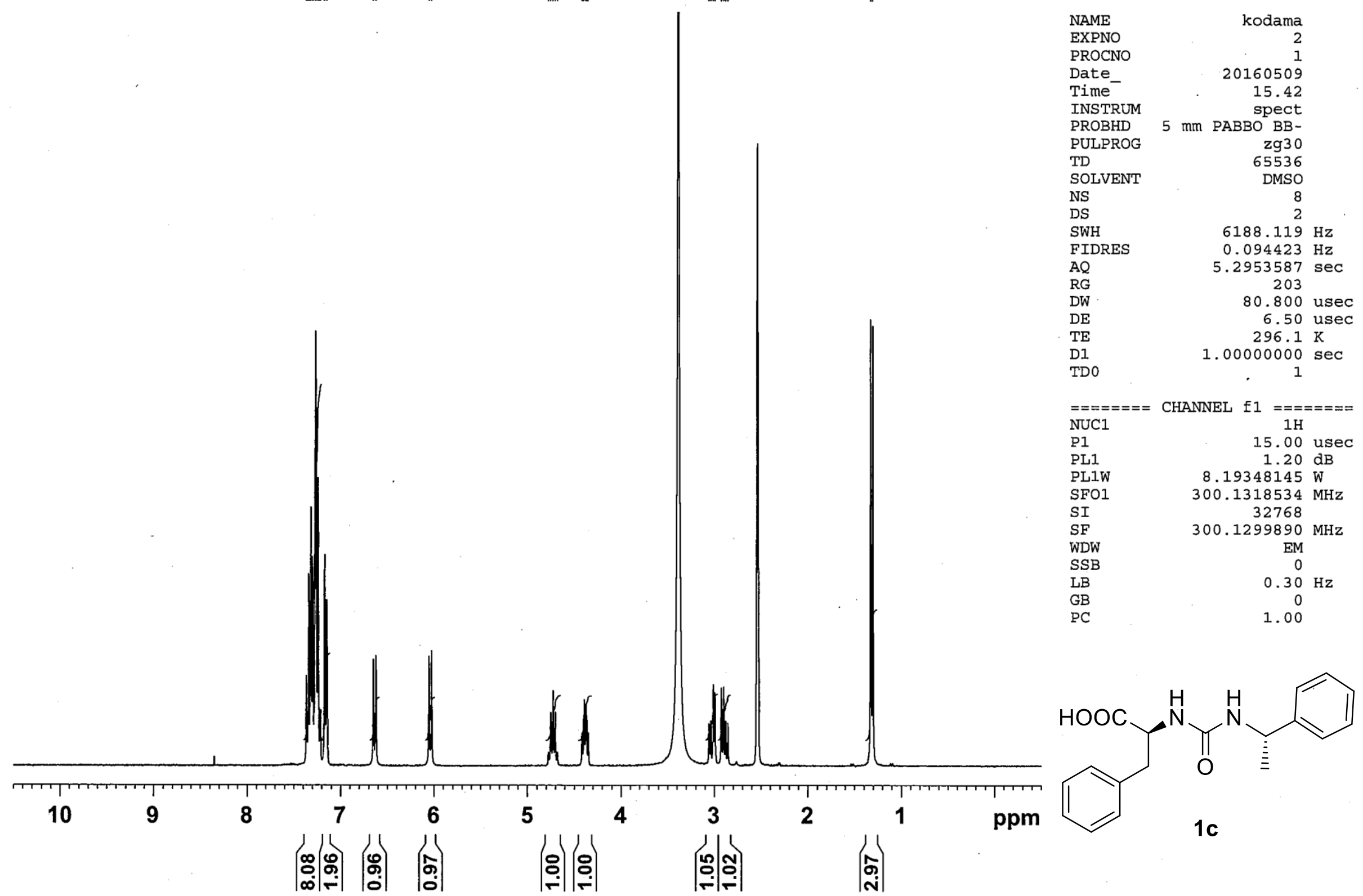


13C with dec. CPQNP

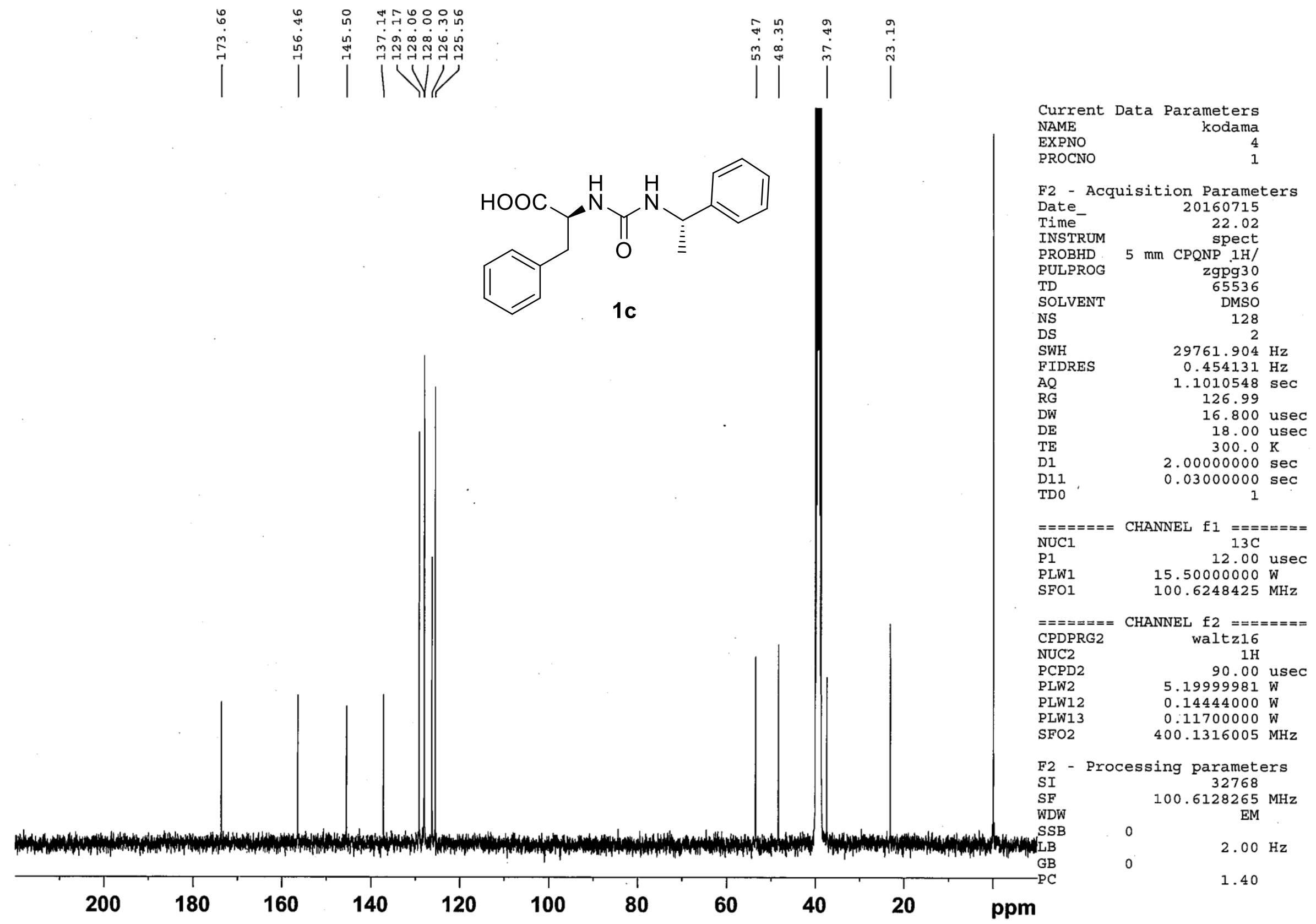




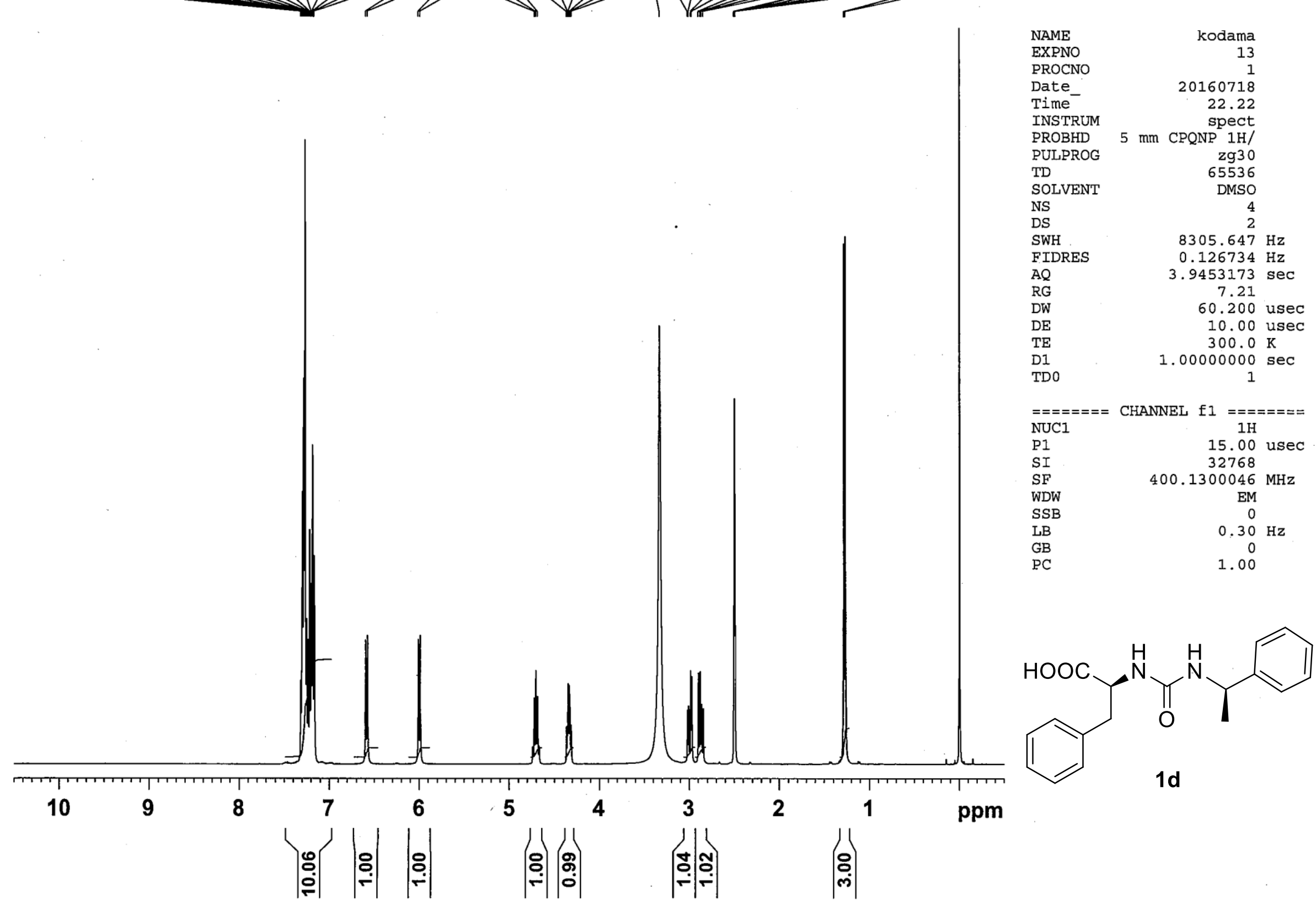


1H CPQNP

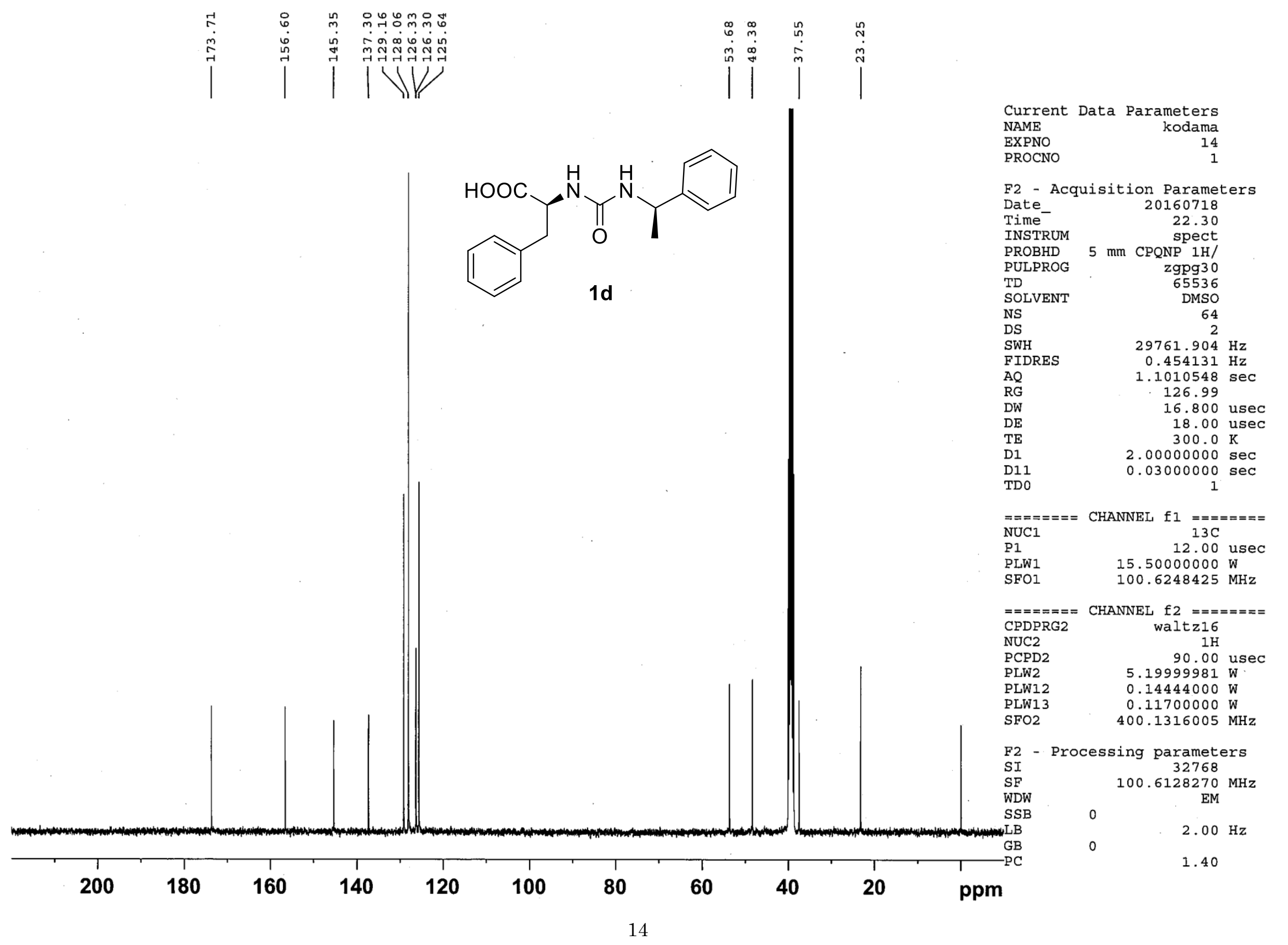




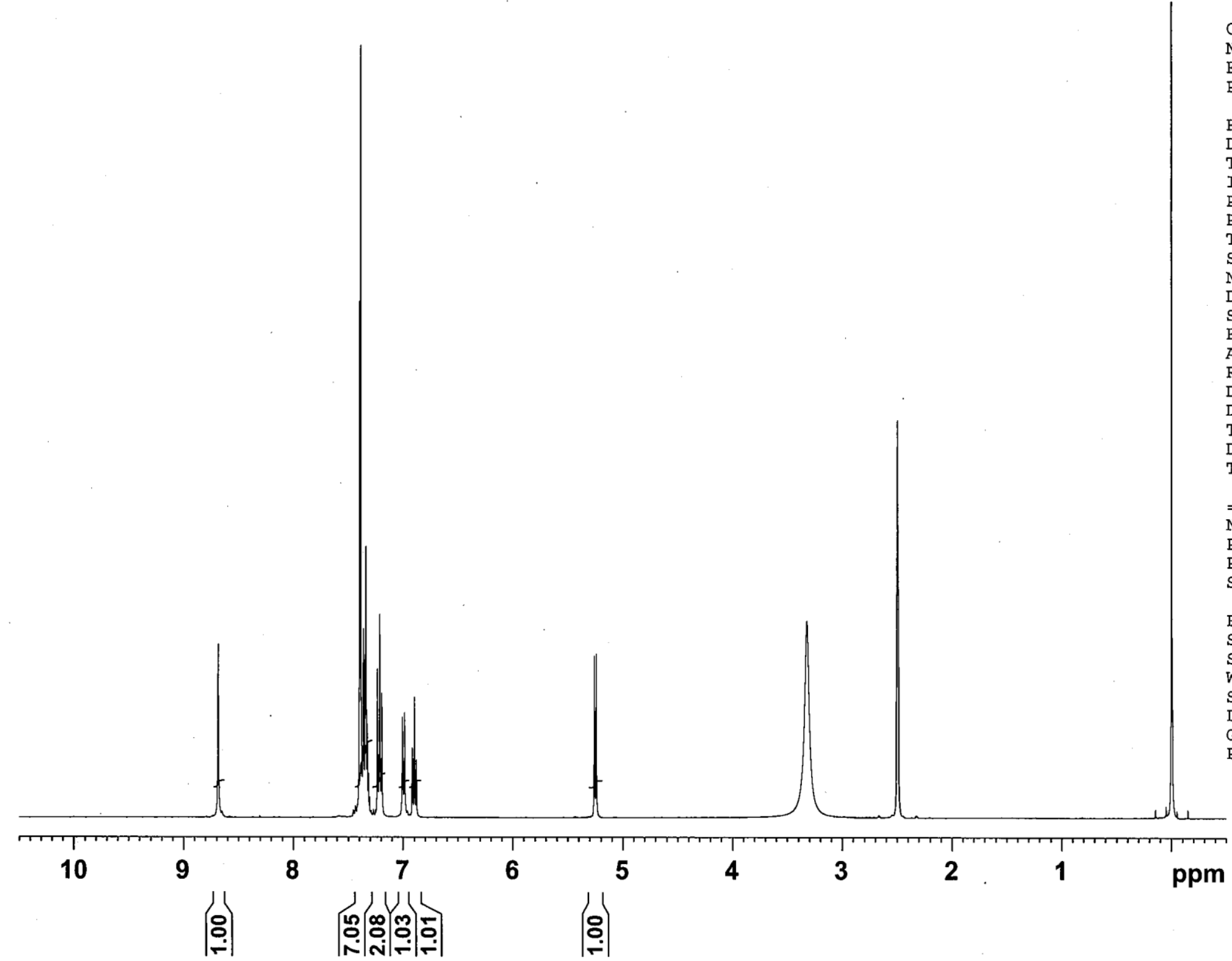

$\begin{array}{lr}\text { Current Data } & \text { Parameters } \\ \text { NAME } & \text { kodama } \\ \text { EXPNO } & 24\end{array}$

NAME

PROCNO

F2 - Acquisition Parameters

$\begin{array}{lr}\text { Date_ } & 20160720 \\ \text { Time } & 13.55\end{array}$

Time

INSTRUM spect

PROBHD $5 \mathrm{~mm}$ CPQNP 1H/

PULPROG

TD

65536
DMSO

SOLVENT

NS

SWH

FIDRES

$A Q$

RG

DE

TE

D1

TDO

8305.647 Hz

$0.126734 \mathrm{~Hz}$

3.9453173 sec

12.9

60.200 usec

10.00 usec

$300.0 \mathrm{~K}$

$1.00000000 \mathrm{sec}$

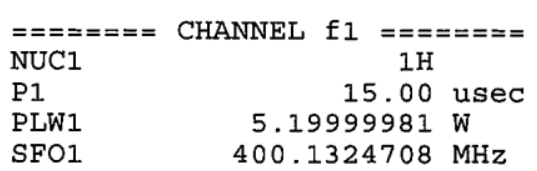

SFO1 $400.1324708 \mathrm{MHz}$

F2 - Processing parameters
SI

$\begin{array}{lc}\text { SF } & 400.1300043 \mathrm{MHz} \\ \text { WDW } & \text { EM }\end{array}$

SSB $\quad 0$

$\begin{array}{lll}\mathrm{LB} & 0.30 \mathrm{~Hz}\end{array}$

$\begin{array}{ll}\text { PC } & 0\end{array}$

HOOC

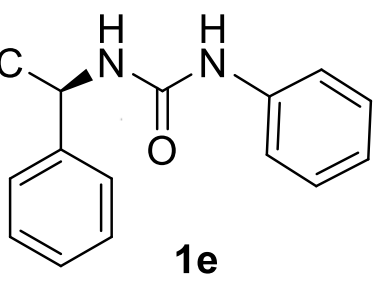


13C with dec. CPQNP

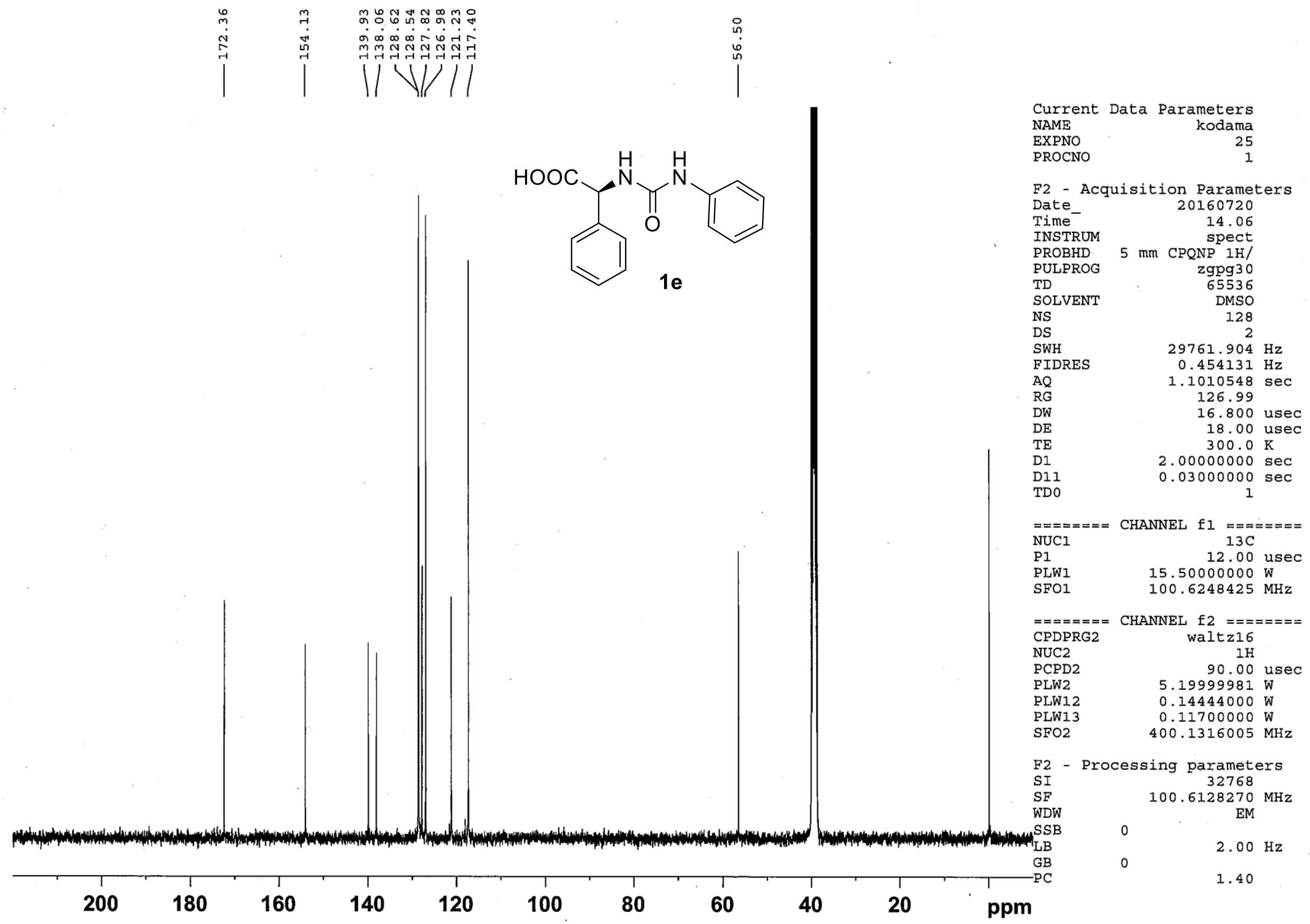




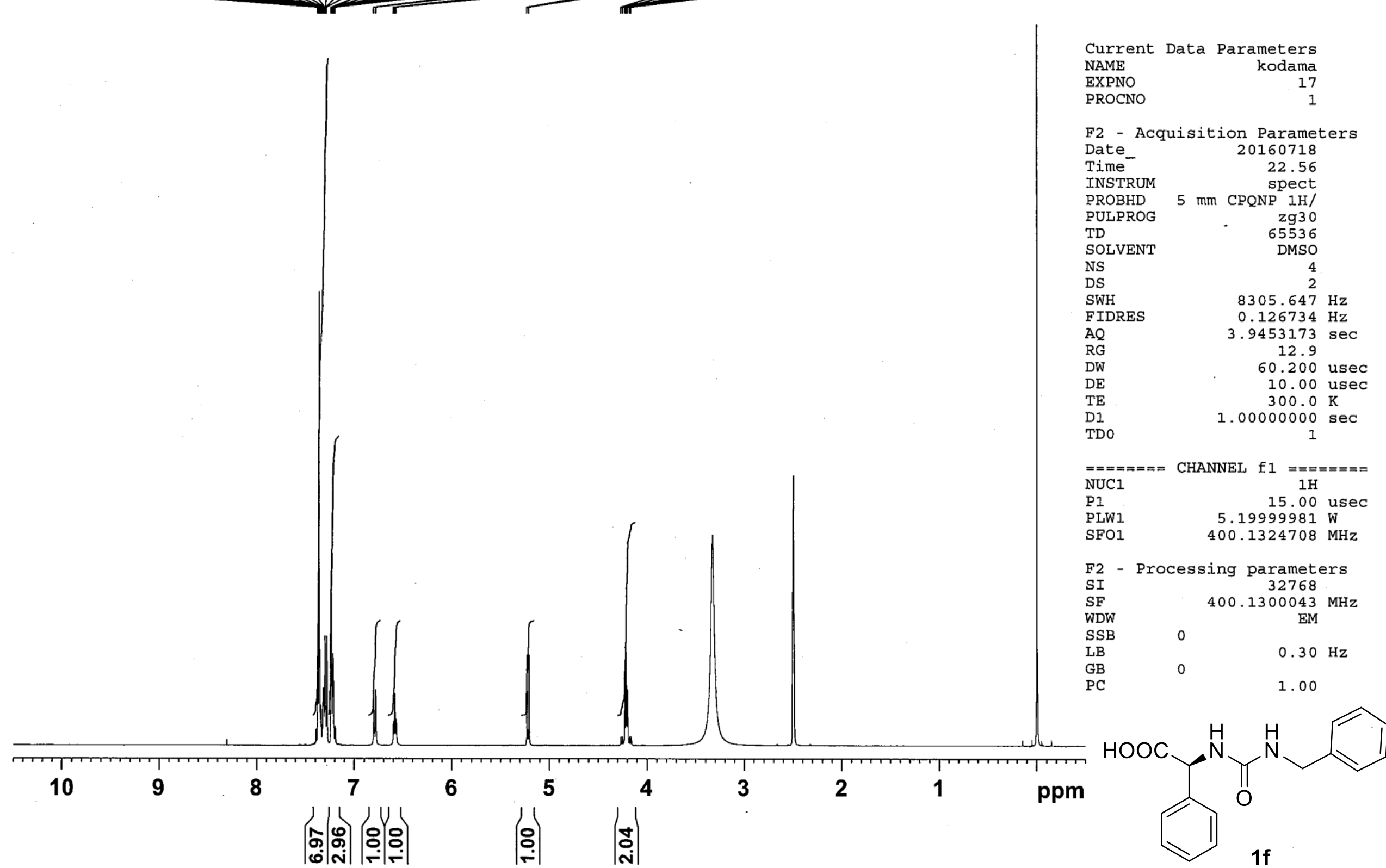


13C with dec. CPQNP

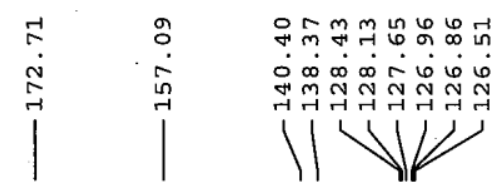

( )

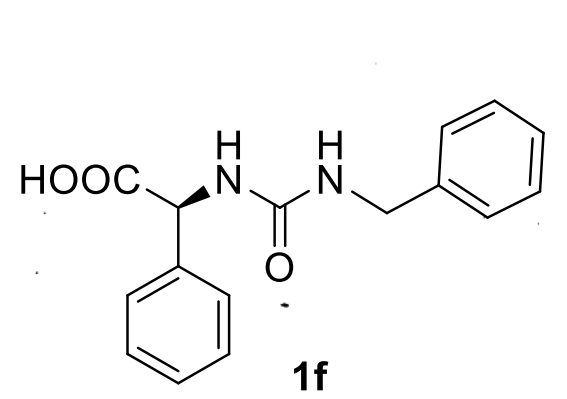

Current Data Parameters

$$
\text { EXPNO }
$$

kodama
18

$$
1
$$

F2 - Acquisition Parameters

Date__ 20160718

Time 23.04

$5 \mathrm{~mm}$ CRQNP $1 \mathrm{H} /$

PUIRO 5 mm CPQNP 1 H/

zgpg 30

TDITENT

NS

DS

$\begin{array}{lr}\text { SWH } & 29761.904 \mathrm{~Hz} \\ \text { FIDRES } & 0.454131 \mathrm{~Hz}\end{array}$

AO $\quad 0.454131 \mathrm{~Hz}$

RG $\quad 126.99$

DW $\quad 16.800$ usec

DE $\quad 18.00$ usec

D1

$300.0 \mathrm{~K}$

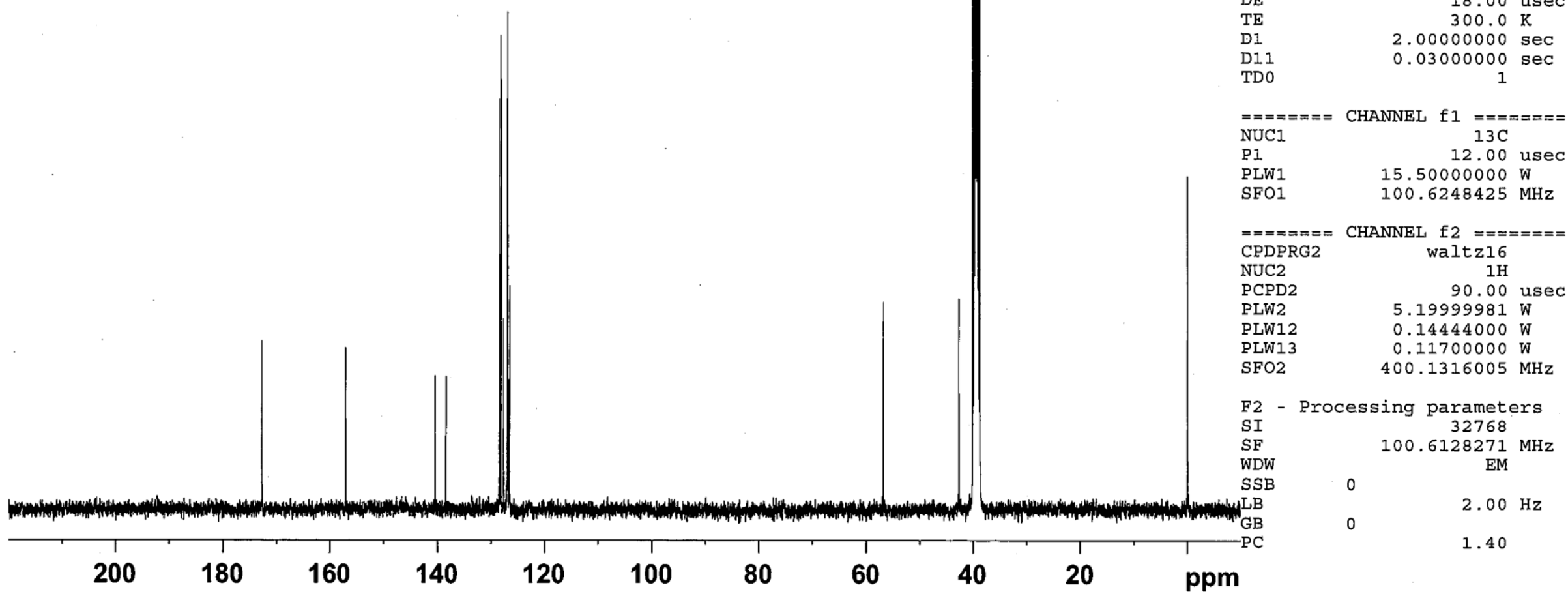




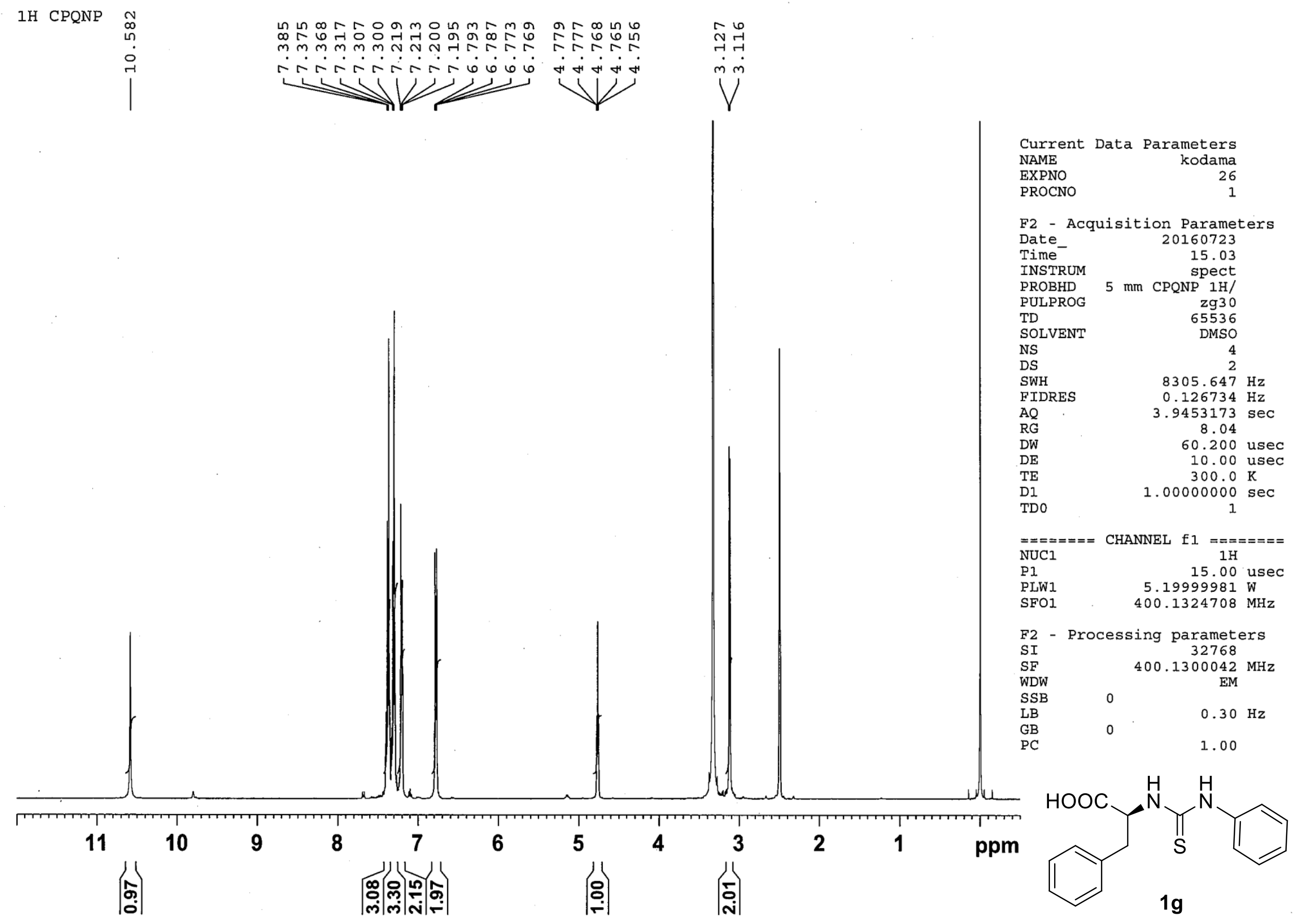


13C with dec. CPQNP

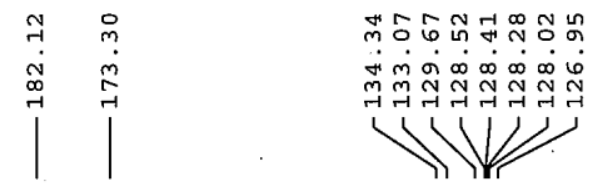

|

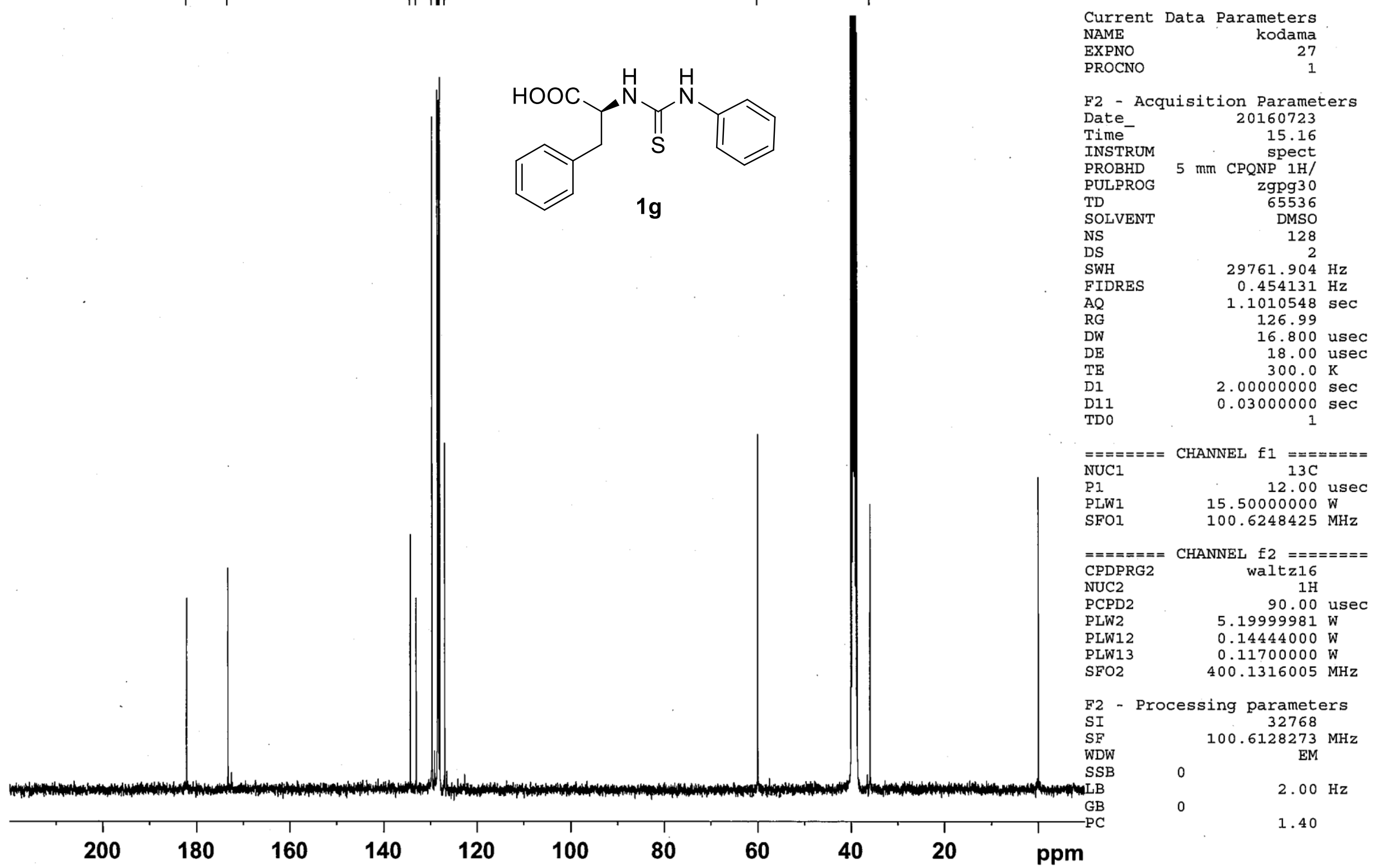




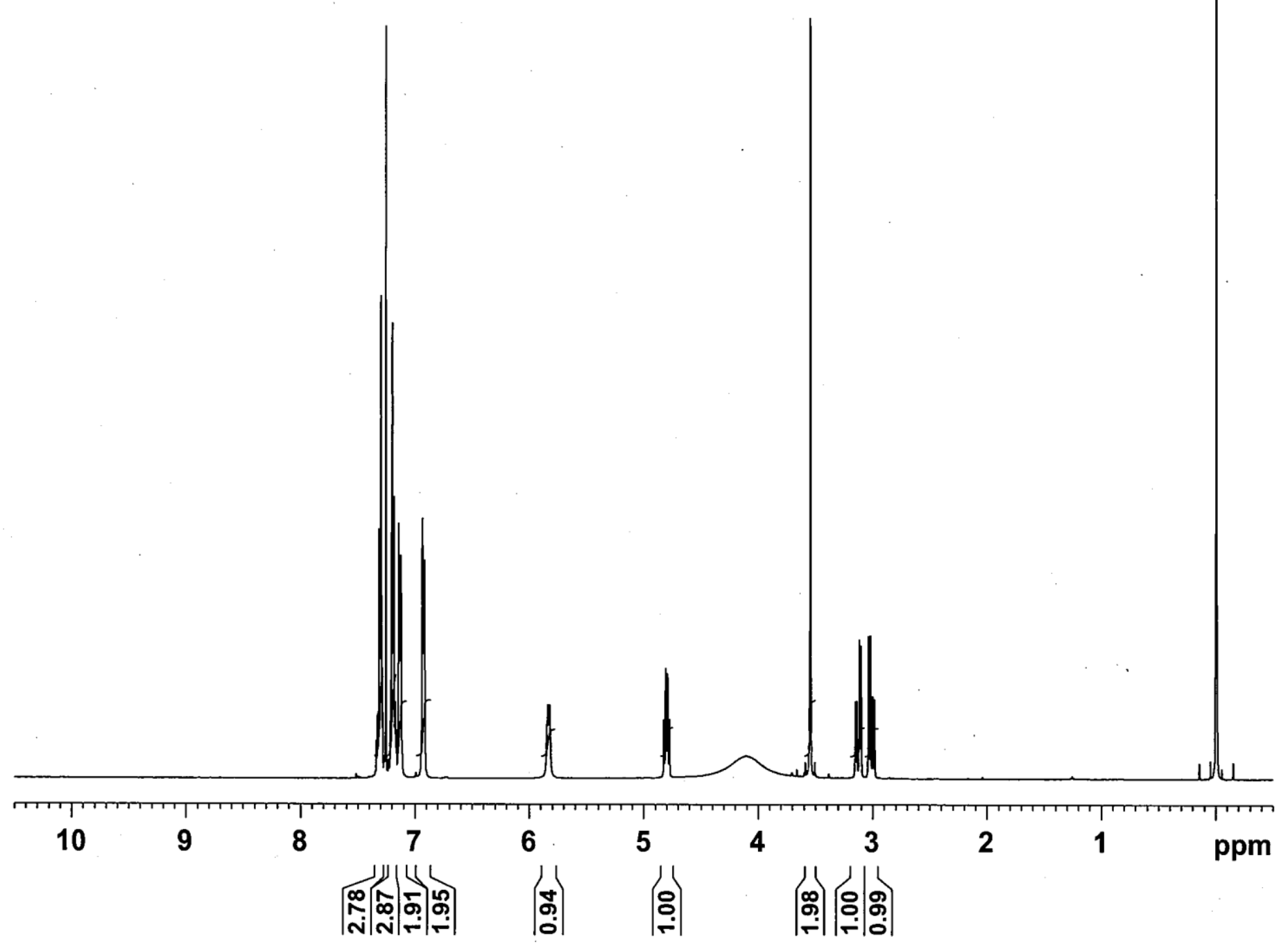

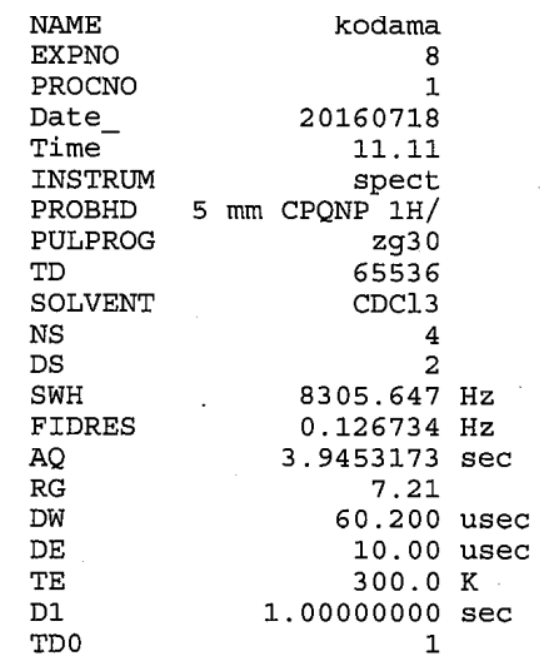

$== \pm====$ CHANNEL fl $==x====0$

NUC1

P1

SI

WDW

SSB

$L B$

GB

$1 \mathrm{H}$

15.00 usec

$400.1300109 \mathrm{MHz}$

$\mathrm{EM}$
0
$0.30 \mathrm{~Hz}$

$0.30 \mathrm{~Hz}$

1.00

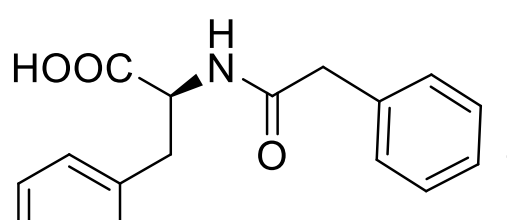

$1 \mathrm{~h}$ 
$13 \mathrm{C}$ with dec. CPQNP

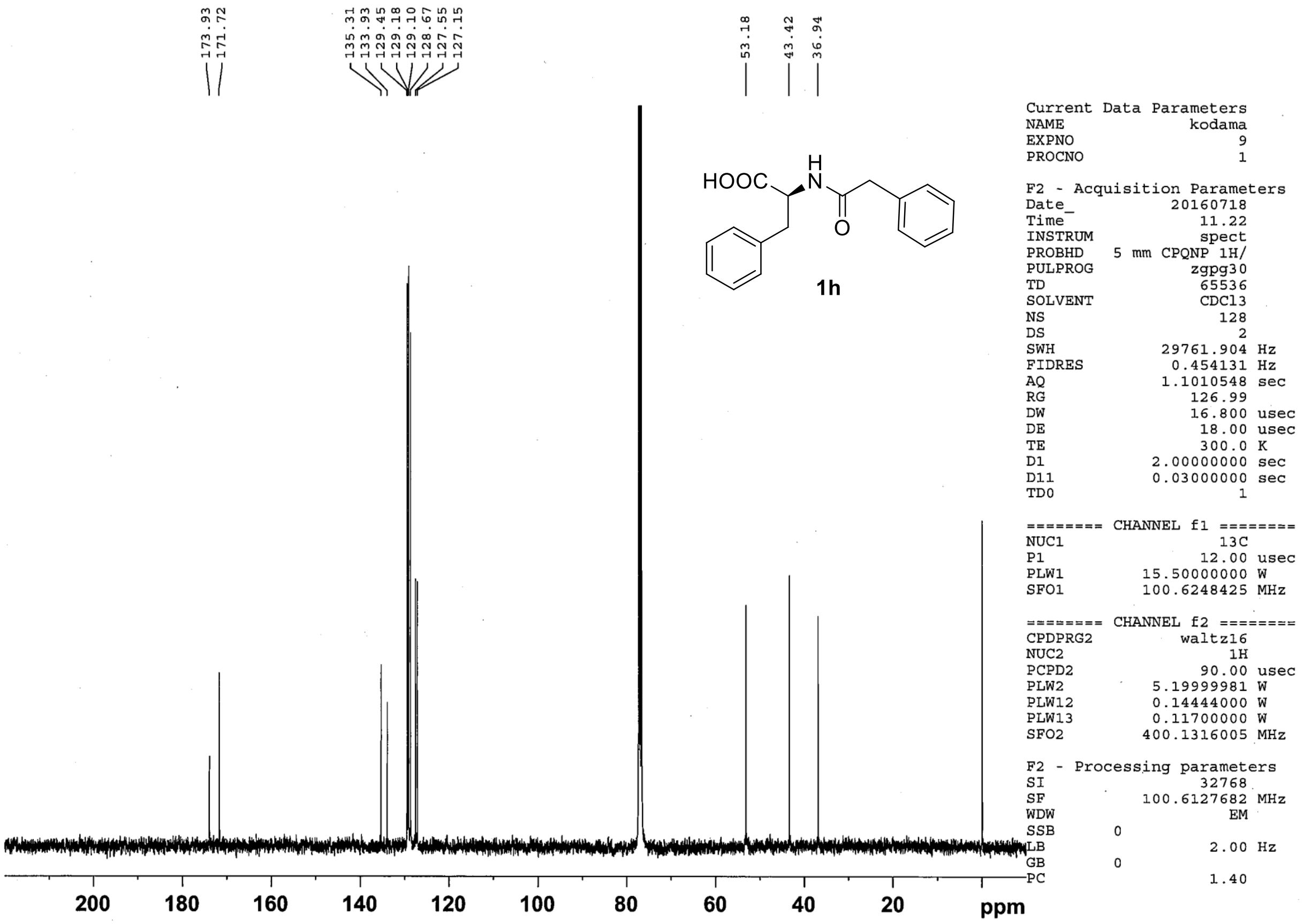

